\title{
Variabilidad intraestacional de la precipitación en Colombia y su relación con la oscilación de Madden-Julian
}

\author{
Claudia Elizabeth Torres-Pineda, José Daniel Pabón-Caicedo*
}

Grupo de investigación “Tiempo, clima y sociedad”, Departamento de Geografía, Universidad Nacional de Colombia, Bogotá, Colombia

\begin{abstract}
Resumen
Se exploró el efecto de la oscilación de Madden-Julian (OMJ) en la precipitación en diferentes regiones del territorio colombiano. Mediante la comparación del índice de Madden-Julian (IMJ) y de los índices de precipitación (IP), y el uso de coeficientes de correlación y comparación de espectros, se analizó la relación entre la OMJ y la variabilidad intraestacional de la precipitación. Se estableció que las fases extremas de la OMJ se relacionaban con anomalías en la precipitación en Colombia; sin embargo, en el análisis de correlación se obtuvieron coeficientes muy bajos, a pesar de que los espectros de las series del IMJ y de los IP evidenciaron una señal clara en el período de los 30 días. En la comparación de la respuesta de los IP a las fases extremas de la OMJ, expresadas en los valores máximos y mínimos de los IMJ sobre la longitud de $120^{\circ} \mathrm{O}$, se estableció que en los extremos positivos de dicho índice (fase positiva o 'subsidente') se registraba una disminución de la precipitación pentadal en algunas regiones del país, en tanto que en los valores extremos negativos (fase negativa o convectiva) la precipitación pentadal aumentaba; en siete de las 24 regiones del país no se pudo determinar un patrón de respuesta definido. Con base en estos resultados, se elaboraron dos mapas de respuesta de la precipitación en las regiones del país en las fases extremas de la OMJ (un mapa para la respuesta en los valores mínimos del IMJ y otro mapa para los máximos), los cuales pueden utilizarse en la predicción climática operativa en algunas regiones de Colombia. (C) 2017. Acad. Colomb. Cienc. Ex. Fis. Nat.

Palabras clave: Variabilidad climática intraestacional en Colombia; Oscilación de Madden-Julian, América del Sur; Precipitación en Colombia.
\end{abstract}

Intraseasonal variability of precipitation in Colombia and its relationship with the Madden-Julian oscillation

\begin{abstract}
We explored the effect of the Madden-Julian oscillation (MJO) on the rainfall in different regions of Colombia by comparing the Madden-Julian index (MJI) and precipitation indexes (PI) using correlation coefficients and wavelet spectra comparison. We found that the MJO extreme phases related to precipitation anomalies, but in the correlation analysis we obtained very low coefficients, although the spectra of the series of both MJI and PIs showed a clear signal over the period of 30 days. In comparing the response of IPs to the extreme phases of the MJO, expressed in maximum and minimum values of the $\mathrm{MJI}$ at $120^{\circ} \mathrm{W}$, we established that under the IMJ positive extremes (positive or subsidence phase) pentadal precipitation decreased in some of the regions, while in the negative extremes (negative or convective phase) we registered an increase; in seven of the 24 regions, no response pattern was identified. Based on this finding, we produced two maps of the response of regional rainfall in the MJO extreme phases (a map for the response to MJI minimum values, and another for the maximum values), which could be used for operational climate prediction in some regions of Colombia. (c) 2017. Acad. Colomb. Cienc. Ex. Fis. Nat.
\end{abstract}

Key words: Intraseasonal climate variability in Colombia; Madden-Julian oscillation over South America; Colombian precipitation.

\section{Introducción}

La variabilidad climática se expresa mediante oscilaciones de variables climatológicas en ciclos de meses (intraestacional), años (interanual) y decenios (interdecadal). En el modo interanual el ciclo El Niño-La Niña- Oscilación del Sur (ENOS) tiene un marcado efecto sobre el total de lluvia estacional de una región dada, mientras que la ocurrencia de episodios de lluvias extremas en el transcurso de una estación es modulada por oscilaciones intraestacionales. Las fases extremas de estas últimas trae consigo cambios en la intensidad de eventos extremos (como lluvias torrenciales, desbordamientos) en la estación lluviosa, agudización/suavización transitoria de fenómenos hidroclimáticos como sequías/inundaciones (Pabón, 2011) con lo que afectan de

\footnotetext{
*Correspondencia:

José Daniel Pabón-Caicedo,jdpabonc@unal.edu.co

Recibido: 24 de mayo de 2016

Aceptado: 7 de febrero de 2017
} 
manera recurrente diversos aspectos de la sociedad como las actividades y la producción agrícola, el recurso hídrico y la salud humana, entre otros. Por esta razón, se realizan esfuerzos por conocer a profundidad la causa de estas oscilaciones para mejorar la predicción climática a fin de reducir los impactos negativos que generan.

La predicción climática en el ámbito colombiano se ha basado principalmente en el conocimiento sobre particularidades del ciclo ENOS y la expresión de este en el clima de una región determinada, con lo que se ha tenido cierto éxito en la predicción en escala estacional. No obstante, cuando se ha intentado realizar predicción en resolución intermensual (mes a mes), con frecuencia la predicción presenta fallas. Una fuente de error en la predicción climática mes a mes se asocia a la falta de inclusión del modo intraestacional que, por periodos de semanas o un mes, activa o desactiva las precipitaciones, inicia o culmina antes la estación lluviosa, o la interrumpe o la prolonga.

Desde que Waliser, et al., (2003) plantearon la posibilidad de predicción de las fases de la Oscilación MaddenJulian (OMJ), diversos intentos se han desarrollado para lograr la predicción de fases de variabilidad de la precipitación. En el contexto nacional, con el fin de incluir el modo intraestacional en los esquemas de predicción, diversos investigadores han explorado la relación entre las Oscilaciones Madden-Julian y la variabilidad intraestacional de la precipitación en el ámbito colombiano (Poveda, et al., 2002; Pabón \& Dorado, 2008; Torres-Pineda, 2012; Yepes \& Poveda, 2013). No obstante, la identificación de estrechas relaciones que permitan usar el Indice Madden-Julian (IMJ) como predictor ha tenido resultados poco esperanzadores, dado que en la variabilidad climática intraestacional actúan otros modos diferentes a los generados por la OMJ (Jones \& Schemm, 2000; Lau y Waliser, 2012; Torrres-Pineda, 2012). En el actual estado del conocimiento está pendiente por aislar los modos intraestacionales diferentes a los causados por la OMJ, explicar su origen e identificar la forma como se puedan utilizar junto con el IMJ como predictores en pronóstico climático mensual. La necesidad de incorporar los modos intraestacionales en los esquemas de predicción es aún más aguda en el contexto colombiano.

Diversos autores señalan a las Oscilaciones de MaddenJulian (ondas con período de 30-60 días en la presión y el viento) como uno de los factores responsables de fluctuaciones intraestacionales en la precipitación y la temperatura (Paegle, et al., 2000; Lau \& Waliser, 2012; Donald, et al., 2006; Zhang, 2013; Alvárez, et al., 2016). Estas ondas, al desplazarse sobre el Pacífico tropical de occidente a oriente transitan sobre el norte de Suramérica, en donde se localiza el territorio colombiano, ocasionan fluctuaciones de la precipitación en la escala intraestacional. A pesar de las claras evidencias de la presencia de estas ondas, de las activaciones/desactivaciones que generan en la precipitación y de sus impactos en la región, poco se ha avanzado en la comprensión de la relación OMJ y la variabilidad de la precipitación en diferentes regiones del territorio colombiano, por lo que aún es insuficiente el conocimiento sobre las mismas para elaborar esquemas de predicción de este componente de la variabilidad climática.

Los estudios sobre la variabilidad climática intraestacional tienen su inicio en los años 70 cuando Madden y Julian (Madden \& Julian, 1971) presentaron la primera evidencia de una oscilación de 30-60 días en la presión atmosférica diaria registrada en la estación de la Isla Cantón (Madden \& Julian, 1971), hecho por el que este tipo de oscilaciones también se conocen como oscilación MaddenJulian. El análisis original de (Madden \& Julian, 1971) sugiere que las características principales de esta oscilación son su propagación hacia el este desde el Océano Indico y su escala temporal entre 40 y 55 días. Desde entonces hasta el presente, cantidad de estudios han confirmado la existencia de diferentes modos de variabilidad intraestacional en diversas variables climatológicas e intentado identificar las causas con miras a establecer predictores en esta escala. Un resumen de la exploración en este tema específico se encuentra en (Lau \& Waliser, 2012). Diversos autores han estudiado las oscilaciones intraestacionales en otras variables como la precipitación de diferentes regiones geográficas del mundo. Krishnamurti \& Shukla, 2007 encontraron modos de 45 y 20 días en la precipitación en la India; por su parte Wang \& Zhang (1996) exploraron la variabilidad intraestacional de precipitación en China y encontraron periodos de 21 y 43 días. Un análisis realizado también para África (Janicot \& Sultan, 2001) permitió identificar señales por 10-25 y 25-60 días en la convección y la precipitación en el Oeste de la región. Además, Ye \& Cho (2001) analizaron datos de la precipitación para Estados Unidos y encontraron señales de 24 y 37 días.

Para Sur América Carvalho \& Jones (2004) determinaron que la OMJ modula la intensidad de la Zona de Confluencia Intertropical Suramericana (SACZ) con la persistencia de episodios de precipitaciones abundantes de más de tres días; adicionalmente, encontraron que la fase de supresión de actividad convectiva sobre Indonesia y de incremento en el Pacífico central, aumenta un 95 por ciento la precipitación diaria sobre el norte y noreste de Brasil, mientras que, con las características opuestas se observa un incremento de la convección sobre Indonesia y una disminución sobre el Pacífico central. Igualmente, Martin \& Schumacher (2012), basados en 12 años de precipitación diaria de satélite y vientos de reanálisis, realizaron un estudio sobre la modulación de la OMJ para el Caribe. Clasificaron las anomalías de precipitación en ocho fases de la siguiente manera: fases 1 y 2 (cuando la fase convectiva de la OMJ se encuentra en África y el Océano Índico occidental), las fases de 3 y 4 (cuando la fase convectiva de la OMJ está en el Océano Índico y el Continente Marítimo), las fases 5 y 6 (cuando la fase convectiva de la OMJ está en el Pacífico occidental), y las fases 7 y 8 cuando la fase convectiva de la OMJ está en el hemisferio occidental). Concluyeron que 
la variabilidad intraestacional es mayor durante septiembrenoviembre (SON), aunque, alguna modulación de la precipitación aparece por la OMJ en todas las estaciones. Además, observaron anomalías de precipitación de hasta $50 \%$ por encima (por debajo) de la media anual en las fases 1 y 2 (5 y 6) de la OMJ. En estos casos, las anomalías en los vientos actúan para disminuir (aumentar) la fuerza de los vientos alisios predominantes del Este. Igualmente, la región del chorro del Caribe de bajo nivel (CLLJ) también es sujeta a la variabilidad intraestacional, y su magnitud varía con la fase de la OMJ. Así, la variabilidad intraestacional en el CLLJ asociado a la OMJ se observa en todas las estaciones y muestra una coherencia significativa con la variabilidad en la precipitación. Por último, eventos extremos de precipitación sobre las islas del Caribe mostraron una fuerte relación con la fase de OMJ, siendo más frecuente en las fases 1 y 2 de un evento OMJ. Del reciente trabajo publicado por Álvarez, et al. (2016), quienes analizaron el efecto de las diferentes fases de la oscilación Madden-Julian sobre la temperatura y la precipitación de Sur América apoyados en el índice multivariado RMM de Wheler-Hendon, se puede ver que las fases 1 y 8 (convección sobre el hemisferio occidental y África) generan sobre la región anomalías en diferentes variables; sobre el noroccidente de América del sur, donde se localiza el territorio colombiano, estas fases de la OMJ generan las anomalías que se exponen en la tabla 1.
Para el norte de Suramérica se han encontrado señales con periodos de 25-30 y de 50-60 días. Poveda, et al. (2002) evaluaron el comportamiento a escala diaria y horaria de la precipitación en los Andes tropicales de Colombia y su relación con la oscilación de Madden-Julian y concluyeron que esta cambia la amplitud del ciclo diurno de precipitación en la región estudiada, pero no cambia su fase, lo que señala que la oscilación de 30-60 días es otro modulador macroescalar del ciclo diurno que afecta la hidrología de los Andes tropicales colombianos. Años más tarde, con base en registros de precipitación media de cada diez días tomados de estaciones de la región Norte de Sur América y del Caribe, Dorado (2007) y Pabón \& Dorado (2008) analizaron la variabilidad intraestacional de la precipitación de la región; el análisis espectral de un índice de precipitación decadal (DPI) permitió identificar señales con periodos entre 20-25, 30,40 y 50-60 días, las cuales estuvieron bien definidas en la región Caribe y en algunos lugares de los Andes; las señal con período de 60 días se presentó solamente en la región Caribe y en algunos lugares del Pacífico; en las cuencas de la Orinoquía y el Amazonas las señales de estas distribuciones no se encuentran claramente definidas. Pabón \& Dorado (2008) también realizaron el análisis de correlación entre la variabilidad intraestacional regional y la oscilación MaddenJulián pero el coeficiente de correlación encontrado fue muy bajo debido a la presencia de señales diferentes a las

Tabla 1. Anomalías generadas en diferentes períodos del año por las fases 1 y 8 de la Oscilación Madden-Julian, identificada según el índice RMM, sobre el noroccidente de Sudamérica

\begin{tabular}{|c|c|c|c|c|}
\hline & DEF & MAM & JJA & SON \\
\hline $\begin{array}{l}\text { Anomalía de altura } \\
\text { de geopotencial }\end{array}$ & No marcadas & No marcadas & No marcadas & No marcadas \\
\hline $\begin{array}{l}\text { Anomalías de } \\
\text { velocidad otencial }\end{array}$ & Negativas & Negativas & Negativas & Negativas \\
\hline $\begin{array}{l}\text { Anomalías de } \\
\text { temperatura del aire } \\
\text { en superficie }\end{array}$ & $\begin{array}{l}\text { Negativas (hasta de }-0,2^{\circ} \mathrm{C} \text { ) } \\
\text { en el sector occidental } \\
\text { (Pacífico colombiano), más } \\
\text { marcado (hasta } 0,4^{\circ} \mathrm{C} \text { ) en el } \\
\text { norte; positivas }\left(0,2^{\circ} \mathrm{C} \text { ) en }\right. \\
\text { el sector sur y suroriental, } \\
\text { más marcadas }\left(0,4^{\circ} \mathrm{C}\right) \text { en } \\
\text { la Amazonía; cercano a lo } \\
\text { normal sobre la Orinoquía, } \\
\text { nororiente y Caribe }\end{array}$ & $\begin{array}{l}\text { En el suroccidente del } \\
\text { Caribe negativas muy } \\
\left.\text { marcadas (hasta }-0,5^{\circ} \mathrm{C}\right) \text {; } \\
\text { en la parte andina negativas } \\
\left(-0,2^{\circ} \mathrm{C}\right) \text {; sobre la Orinoquía } \\
\text { positivas }\left(0.2^{\circ} \mathrm{C}\right) \text {, pero muy } \\
\left.\text { altas (hasta } 0,5^{\circ} \mathrm{C}\right) \text { sobre la } \\
\text { Amazonia Perú-Ecuador- } \\
\text { Colombia. }\end{array}$ & $\begin{array}{l}\text { Pacífico colombiano } \\
\text { de ligeras anomalías a } \\
-0,2^{\circ} \mathrm{C} \text {; sobre el Caribe } \\
\text { anomalías negativas de } \\
\text { hasta }-0.4^{\circ} \mathrm{C} \text {; Amazonía, } \\
\text { positivas con el núcleo de } \\
\text { hasta } 0,4^{\circ} \mathrm{C} \text {; resto del país } \\
\text { sin anomalías }\end{array}$ & $\begin{array}{l}\text { Anomalías de }-0,3^{\circ} \mathrm{C} \\
\text { a } 0.4^{\circ} \mathrm{C} \text { sobre Caribe } \\
\text { y Pacífico; región } \\
\text { Andina, Orinoquía } \\
\text { y noroccidente de } \\
\text { la Amazonia con } \\
\text {-0,3; suroriente de la } \\
\text { Amazonia con } 0,3^{\circ} \mathrm{C}\end{array}$ \\
\hline $\begin{array}{l}\text { Anomalías de } \\
\text { precipitación (la } \\
\text { probabilidad de } \\
\text { que el acumulado } \\
\text { semanal exceda el } \\
\text { tercer tercil) }\end{array}$ & $\begin{array}{l}\text { Excesos en el sur y centro } \\
\text { (Eje Cafetero) de la región } \\
\text { Andina y en el sector } \\
\text { central (Valle del Cauca) } \\
\text { de la región Pacífica }\end{array}$ & $\begin{array}{l}\text { Alta probabilidad de exceso } \\
\text { sobre Nariño y Cauca; } \\
\text { probabilidad de exceder en } \\
\text { el Pacífico, Eje Cafetero, } \\
\text { montaña antioqueña, centro } \\
\text { de la región-litoral Caribe } \\
\text { y sobre el Archipiélago de } \\
\text { San Andrés y Providencia }\end{array}$ & $\begin{array}{l}\text { Incremento marcado } \\
\text { (especialmente bajo fase } \\
\text { 1) de la probabilidad de } \\
\text { exceder el tercer tercil } \\
\text { sobre toda la región } \\
\text { Andina y Caribe, incluido } \\
\text { el Archipiélago de San } \\
\text { Andrés y Providencia }\end{array}$ & $\begin{array}{l}\text { Alta probabilidad de } \\
\text { exceder el tercer tercil } \\
\text { en el sur de la región } \\
\text { Pacífica, región Andina } \\
\text { y Caribe. Muy alta } \\
\text { sobre Antioquia y } \\
\text { litoral Caribe central } \\
\text { bajo la fase } 8 \text {. }\end{array}$ \\
\hline
\end{tabular}

DEF - Diciembre-Enero-Febrero; MAM -Marzo-Abril-Mayo; JJA -Junio-Julio-Agosto; SON - Septiembre-Octubre-Noviembre (información extractada para la región a partir de los mapas de Álvarez et al., 2015) 
de 30-60 días. Considerando esta situación se utilizó una frecuencia más leve con las series DPI, encontrando una correlación relativamente alta entre periodos depurados de DPI y el indicador Madden-Julián (IMJ). En un estudio más reciente (Trickey, et al., 2012), evaluaron la posibilidad de utilizar la oscilación Madden-Julian en la predicción de la precipitación de la costa ecuatoriana, con base en la elaboración de un indicador que integra el Índice Niño 3 y el Índice Madden-Julian; dicho índice integrado se correlacionó con la precipitación mensual de diferentes estaciones de la región. Sin embargo los resultados obtenidos no permitieron obtener una mejora en la predicción comparada con lo que se hacía con el esquema de predicción estacional ya existente, aunque mencionan haber obtenido algo de mejora para el final de la estación lluviosa. Al parecer, esto ha ocurrido debido a que en la variabilidad de la precipitación representada en series de datos mensuales está integrado el efecto de dos procesos de diversa escala: una interanual (el ciclo ENOS) y otra intraestacional (Oscilación MaddenJulian). La integración de los dos procesos considerados como la causa en un mismo indicador, resulta poco consistente con esta realidad; se debería tratar por aparte los efectos y correlacionarlos con la causa correspondiente, toda vez que la señal de los dos procesos causantes se integran en la variabilidad de la precipitación (proceso efecto) más no en los procesos causa. Otra posible razón es la utilización de datos mensuales para tratar de incluir procesos de 30 60 días. En efecto, Cuadros (2010) en un estudio sobre las características y la relación de la OMJ con las temperaturas superficiales extremas en las regiones de Colombia durante el periodo 1978-2008, determinó con análisis espectral que las oscilaciones intra-estacionales están encubiertas por ondas de mayor energía (de mayor escala temporal con las asociadas al ciclo ENOS), pero las OMJ hacen un aporte significativo a la varianza de las series de temperatura. Torres-Pineda (2012), con análisis espectral (espectro de onditas) a series de precipitación pentadal (acumulados de cinco días), basadas en datos provenientes de mediciones en pluviómetros, encontró señales entre 10-20 días, 30-40 días, 55-60 días, semestrales, interanuales e interdecadales; igualmente identificó que en la escala intraestacional de la precipitación la señal más marcada es la cercana a los 30 días, por lo que intentó relacionar directamente esta señal con la correspondiente en la serie del Indice de Madden-Julian, identificando correlaciones relativamente destacadas para algunas de las regiones analizadas. Yepes \& Poveda (2013), con base en series de precipitación pentadal, provenientes de uno de los niveles de datos obtenidos por medición remota de la Tropical Rain Measurement Mission (TRMM), y con el uso de espectro de Hilbert-Huang, encontraron también una señal importante sobre los 20 días que se expresa mejor sobre la Amazonía y el Pacífico colombiano.

En resumen, durante los últimos dos decenios en el ámbito colombiano ha habido un notable progreso en la identificación de diversos modos intraestacionales en la variabilidad de la precipitación y en el análisis de la relación de estos con las OMJ, pero aún es necesario establecer más claramente tal relación para fundamentar mejor los esquemas de predicción climática en esta escala temporal. Dada esta necesidad, se abordó el trabajo cuyos resultados se exponen a continuación, el cual tuvo como objetivo, además de fortalecer el conocimiento acerca de los diferentes modos de la variabilidad intraestacional de la precipitación de diferentes regiones del territorio colombiano, explorar a mayor profundidad la relación de éstos con la OMJ para generar elementos de predicción climática en la escala intermensual para tales regiones.

\section{Materiales y métodos}

La exploración se efectuó comparando el comportamiento de la variable precipitación y la velocidad vertical en 500 hPa, con el comportamiento del índice Madden-Julian basado en la velocidad potencial en $200 \mathrm{hPa}$.

Para la precipitación se emplearon datos diarios del periodo 1978-2008 de 24 estaciones operadas por el Instituto de Hidrología Meteorología y Estudios Ambientales IDEAM y distribuidas sobre las regiones hidroclimáticas demarcadas por Hurtado (2000) para el territorio colombiano (ver Figura 1 y Tabla 2). La selección de las estaciones meteorológicas se realizó teniendo en cuenta la longitud de la serie y la calidad de la información, así como una distribución espacial que cubriera la mayor cantidad de regiones climáticas señaladas en la figura 1. La lista de estaciones utilizadas para

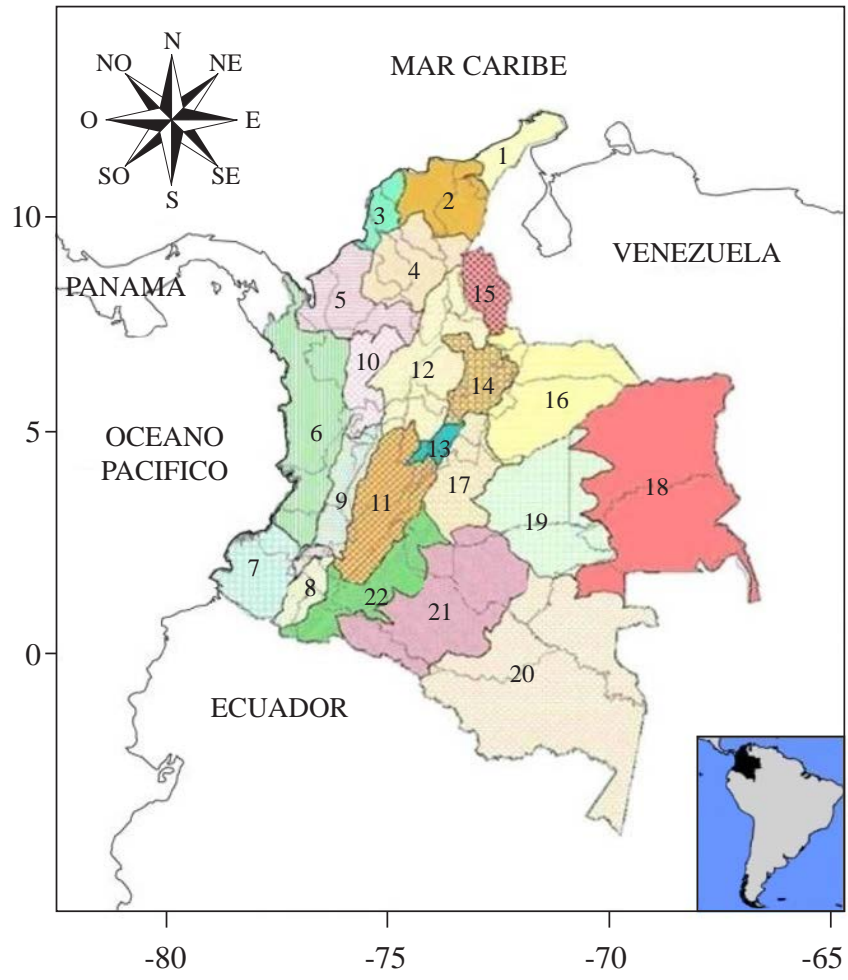

Figura 1. Regiones hidroclimáticas de Colombia analizadas (Fuente: Hurtado, 2000) 
Tabla 2. Denominación de las regiones hidroclimáticas de Colombia (según Hurtado, 2000)

\begin{tabular}{|c|c|}
\hline $\begin{array}{l}\text { No. En el } \\
\text { mapa de la } \\
\text { figura } 1\end{array}$ & Región \\
\hline & Caribe \\
\hline 1 & Alta Guajira \\
\hline 2 & $\begin{array}{l}\text { Noroccidente de la Sierra Nevada de Santa Marta } \\
\text { y cuenca del río Cesar }\end{array}$ \\
\hline 3 & Litoral central \\
\hline 4 & Bajo Magdalena \\
\hline \multirow[t]{2}{*}{5} & Cuenca del río Sinú, Bajo Nechí, región de Urabá \\
\hline & Pacífica \\
\hline 6 & Pacífico norte y centro \\
\hline 7 & Pacífico sur \\
\hline \multirow[t]{2}{*}{8} & Cuenca alta del río Patía \\
\hline & Andina \\
\hline 9 & Cuenca alta del río Cauca \\
\hline 10 & Cuenca media del río Cauca \\
\hline 11 & Cuenca alta del río Magdalena \\
\hline 12 & Cuenca media del río Magdalena \\
\hline 13 & Cuenca del río Bogotá, Sabana de Bogotá \\
\hline 14 & Cuenca del río Sogamoso \\
\hline 15 & Cuenca del río Catatumbo \\
\hline \multirow[t]{2}{*}{23} & Montaña nariñense \\
\hline & Orinoquía \\
\hline 16 & Cuenca del río Arauca \\
\hline 17 & Piedemonte llanero \\
\hline 18 & Nororiente de la Orinoquía \\
\hline \multirow[t]{2}{*}{19} & Orinoquía central \\
\hline & Amazonia \\
\hline 20 & Suroriente de la Amazonía \\
\hline 21 & Centro de la Amazonía \\
\hline \multirow[t]{2}{*}{22} & Piedemonte amazónico \\
\hline & Área insular Caribe occidental \\
\hline 24 & San Andrés y Providencia \\
\hline
\end{tabular}

el análisis se presenta en la tabla 3. En las series con datos faltantes, cuyo período con vacíos no era extenso se optó por generar los datos ausentes, la cual se realizó con base en los valores registrados en estaciones vecinas que tienen marcada influencia sobre la zona de ubicación de la estación en la que aparece el dato faltante. De estaciones cercana a la del dato faltante, se tomaron 1 o 2 estaciones que se relacionan mejor con la primera y se estableció un factor de relación entre los promedios multianuales de precipitación de estas; así se generó el dato multiplicando dicho factor por la precipitación del mes correspondiente en la estación de apoyo. Después de realizar el control de calidad básico a los datos diarios (verificación y descarte de inconsistencias; análisis de homogeneidad), se construyeron series pentadales, decadales y quincenales (acumulados de 5, 10 y 15 días, respectivamente) desde 1994 hasta 2004, período en el que las series presentaron menos datos faltantes

Posteriormente, se calcularon los respectivos índices pentadales, decadales, y quincenales de precipitación, cada uno de acuerdo con la fórmula:

$$
I P_{i, j}=\frac{P_{i, j}-\overline{P_{j}}}{\sigma_{j}}
$$

Donde: $I P_{i j}$ - índice de precipitación de la pentada o década o quincena $j$ del año $i ; P_{i j}$ - precipitación de la pentada o década o quincena $j$ del año $i$; $-\bar{P}_{i j}$ promedio multianual para la pentada, década o quincena $j$; $\sigma_{j}$ - desviación estándar de la pentada, decada, o quincena $j$.

Los datos de velocidad vertical en $500 \mathrm{hPa}$ se obtuvieron del CISL Research Data Archive (RDA), que contiene salidas operacionales del reanálisis gestionado por la sección de soporte de datos del National Center for Atmospheric Research (NCAR). A través de una solicitud personalizada se adquirió un conjunto de datos de Climate Forecast System Reanalysis (CFSR) de cada 6 horas para un periodo de tiempo desde 1 de enero de 1979 hasta el 31 de marzo de 2011. Los archivos correspondientes se obtuvieron en formato netCDF y fue necesario un script en NCL (Lenguaje de Comandos del NCAR) para extraer los datos de velocidad vertical a 500 hPa de cada una de las coordenadas de las estaciones; con base en datos obtenidos para cada 6 horas, se construyeron series pentadales, decadales y quincenales.

Los datos del índice de la Oscilación Madden-Julian (IMJ) usados en este estudio son los publicados por la NOAA en su página http://www.cpc.noaa.gov/products/precip/CWlink/ daily_mjo_index/proj_norm_order.ascii

Para el análisis se utilizaron los datos del IMJ para $120^{\circ}$ $\mathrm{W}$ y $60^{\circ} \mathrm{W}$. Dado que los datos disponibles no presentan el MJI para una longitud dentro del territorio colombiano, se construyó el IMJ para $80^{\circ} \mathrm{W}$ utilizando interpolación lineal entre el IMJ de $120^{\circ} \mathrm{W}$ y $60^{\circ} \mathrm{W}$ denominándolo IMJ80. La serie producida en pentadas, con 6 datos por cada mes, tiene origen desde el año 1978 hasta la actualidad, de manera que se hacen comparables con las series de cada una de las variables meteorológicas de este estudio de IP y de velocidad vertical en $500 \mathrm{hPa}$. Finalmente, se efectuó la suavización de las series del IMJ con media móvil de tres puntos.

Para establecer la influencia de la MJO en el ciclo de precipitación en el territorio colombiano es necesario determinar si eventos de 30-60 días de frecuencia afectan a las diferentes series y están correlacionadas; además, en caso de que esta señal exista, debe corroborarse si corresponde a la presencia de la MJO. La exploración de la influencia de las ondas de Madden-Julian en la variabilidad intraestacional de la precipitación se realizó a partir del análisis de correlación entre las series del IMJ y del IP e intentando 
Tabla 3. Lista de estaciones meteorológicas/climatológicas utilizadas para el análisis

\begin{tabular}{|c|c|c|c|c|c|c|c|}
\hline LAT $\left(^{\circ}\right)$ & $\operatorname{LON}\left({ }^{\circ}\right)$ & Código & Categoría & $\begin{array}{l}\text { Altitud } \\
\text { (m.s.n.m.) }\end{array}$ & Estación & Departamento & Municipio \\
\hline 12,58 & $-81,72$ & 1701502 & RS & 1 & Sesquicentenario & San Andrés & San Andrés \\
\hline 11,13 & $-74,23$ & 1501505 & SP & 4 & Simón Bolívar & Magdalena & Santa Marta \\
\hline 11.53 & $-72,93$ & 1506501 & SP & 4 & Alm. Padilla & La Guajira & Riohacha \\
\hline 10,88 & $-74,58$ & 2904502 & SP & 14 & E Cortissoz & Atlántico & Soledad \\
\hline 10,43 & $-73,25$ & 2803503 & SP & 138 & Alfonso López & Cesar & Valledupar \\
\hline 10,38 & $-75,53$ & 1401503 & $\mathrm{CP}$ & 1 & Escuela Naval & Bolívar & Cartagena \\
\hline 8,90 & $-75,56$ & 1308502 & $\mathrm{CO}$ & 33 & El Salado & Córdoba & Ciénaga de Oro \\
\hline 7,93 & $-72,51$ & 1601501 & SP & 250 & Camilo Daza & Norte de Santander & Cúcuta \\
\hline 7,02 & $-73,80$ & 2315503 & SP & 126 & Yariguíes & Santander & Barrancabermeja \\
\hline 6,22 & $-75,58$ & 2701507 & SP & 1490 & Olaya Herrera & Antioquia & Medellín \\
\hline 5,56 & $-73,36$ & 2403513 & $\mathrm{CP}$ & 2690 & UPTC & Boyacá & Tunja \\
\hline 4.72 & $-74,15$ & 2120579 & SP & 2547 & Eldorado p1-2 & Bogotá & Bogotá \\
\hline 4,03 & $-74,97$ & 2118503 & $\mathrm{CP}$ & 360 & Guamo & Tolima & Guamo \\
\hline 2,97 & $-75,30$ & 2111502 & SS & 439 & Benito Salas & Huila & Neiva \\
\hline 7,07 & $-70,73$ & 3705501 & SP & 128 & Arauca & Arauca & Arauca \\
\hline 6,18 & $-67,48$ & 3801503 & SP & 50 & Puerto Carreño & Vichada & Puerto Carreño \\
\hline 4,17 & $-73,62$ & 3503502 & SP & 445 & Vanguardia & Meta & Villavicencio \\
\hline 5,70 & $-76,65$ & 1104501 & SP & 53 & El Caraño & Choco & Quibdó \\
\hline 3,85 & $-76,97$ & 5311501 & SP & 14 & Buenaventura & Valle & Buenaventura \\
\hline 2,45 & $-76,60$ & 2603503 & SP & 1757 & GL Valencia & Cauca & Popayán \\
\hline 1,38 & $-77,28$ & 5204502 & SP & 1873 & Antonio Nariño & Nariño & Chachagui \\
\hline 4,06 & $-74,66$ & 4411502 & $\mathrm{AM}$ & 153 & La Tagua & Putumayo & Leguízamo \\
\hline 4,20 & $-69,95$ & 4801501 & SP & 84 & Vasquez Cobo & Amazonas & Leticia \\
\hline 1,60 & $-75,53$ & 4403502 & SS & 244 & G Artunduaga & Caquetá & Florencia \\
\hline
\end{tabular}

Categoría

RS: Estación de radiosonda; SP: Estación sinóptica principal; SS: Sinóptica secundaria; CP: Climatológica principal; CO: Climatológica ordinaria; AM: Agro meteorológica

identificar procesos con frecuencia similar en las dos series con base en análisis espectral. Para el análisis de correlación se utilizó el coeficiente de Pearson, toda vez que los índices IMJ e IP analizados son variables continuas con distribución cercana a la normal, aunque con sesgo positivo; así, la correlación se basó en las series del IMJ pentadal original y en el IMJ suavizado con medias móviles de 3 puntos (tres pentadas) con las series originales del IP pentadal, decadal y quincenal. En la comparación de espectros se utilizó el espectro de onditas (según Torrence \& Compo, 1998) del IMJ y del IP a fin de encontrar señales similares en un mismo intervalo de frecuencia.

Otra opción utilizada en el presente trabajo para explorar la relación entre IMJ y el IP fue el análisis de la respuesta de la precipitación de las regiones a fases extremas de la OMJ. Se tomaron seis eventos máximos y seis mínimos del IMJ y se analizó que reacción había en el IP pentadal. Con base en lo anterior se construyeron tablas y mapas que resumen y permiten visualizar la relación analizada.

Finalmente, los resultados obtenidos en la identificación de la relación IMJ y el IP regional se sintetizaron en mapas para apoyo para la predicción climática basados en la respuesta identificada de la precipitación regional cuando el IMJ en $120^{\circ} \mathrm{W}$ o en $80^{\circ} \mathrm{W}$ sobrepase el valor de 2.0 o esté por debajo de -2.0.

\section{Resultados y discusión}

Una primera aproximación a la relación de las oscilaciones intraestacionales y la precipitación sobre el territorio colombiano se presenta en la figura 2 en que se compara el comportamiento de la anomalía de velocidad potencial sobre el nivel de $200 \mathrm{hPa}$ y la distribución espacial de las anomalías de precipitación mensual sobre el territorio colombiano. Es necesario tener en cuenta que el período analizado agosto/2009 - mayo/2010 estuvo influenciado por la fase negativa del ciclo ENOS generada por un evento La Niña, lo que en términos generales debería originar anomalías positivas de precipitación en gran parte del país. No obstante, en la medida como sobre el norte de Suramérica se registra la fase subsidente de la OMJ, hay una disminución de las anomalías positivas de precipitación e incluso aparición de regiones con anomalías negativas; por el contrario, la aparición de la fase convectiva de la OMJ agudizó la presencia de anomalías positivas sobre el país. 
Anomalías de velocidad potencial sobre los Anomalías de precipitación 200hPa entre $5^{\circ} \mathrm{S}$ y $5^{\circ} \mathrm{N}$ (media móvil 5 días) sobre Colombia

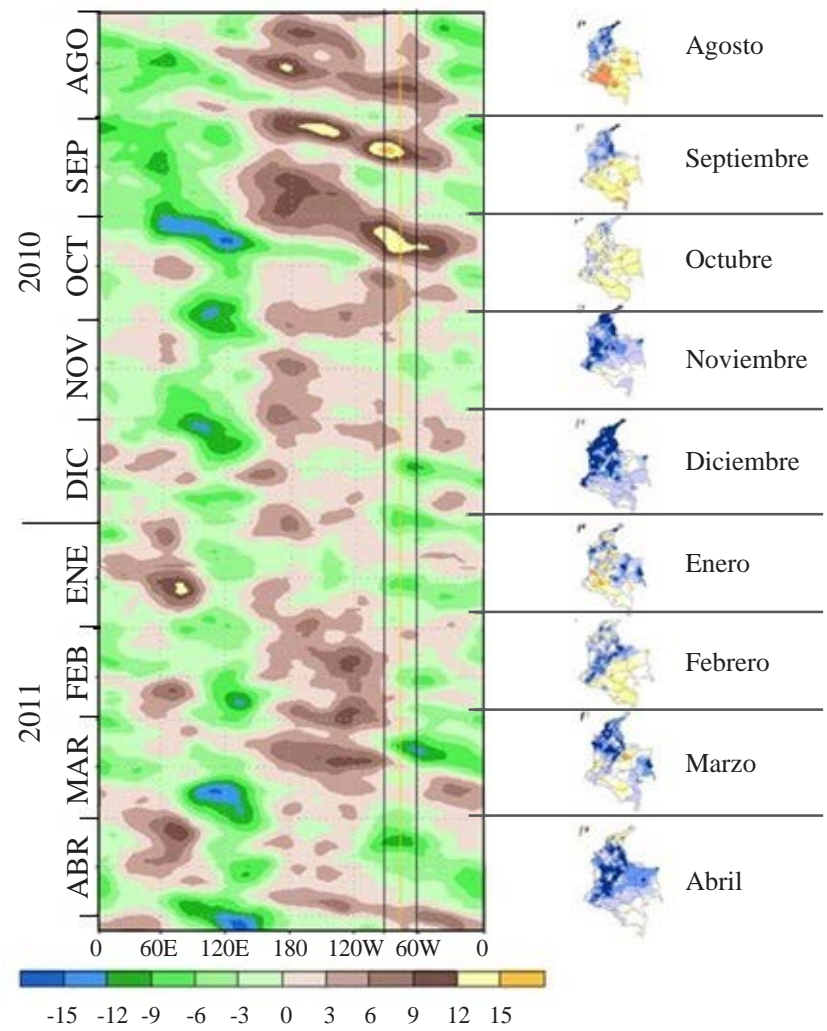

Figura 2. Comparación de comportamiento de la anomalía de velocidad potencial sobre el nivel de $200 \mathrm{hPa}$ y las anomalías de precipitación mensual sobre el territorio colombiano en el período comprendido entre agosto/2010 y mayo/2011. [Los colores azules y verdes (marrón y amarillo) en el gráfico Hovmöller de la izquierda representan valores positivos (negativos) o la fase convectiva (subsidente) de la OMJ; en los mapas de Colombia el color azul (anaranjado y amarillo) presentan lluvias por encima (por debajo) de lo normal. La franja vertical entre líneas negras indica la localización del norte de Suramérica; la línea amarilla muestra los 75W].

En la figura 2, para el primer trimestre presentado, se observan fases subsidentes destacadas de la MJO que coinciden con anomalías negativas de la precipitación especialmente en el Centro y Sur del territorio colombiano; por el contrario, los mapas de anomalías de la precipitación de noviembre, diciembre y abril donde predominan las anomalías positivas sobre gran parte del país concuerdan con fases convectivas de la MJO. Este seguimiento visual también conduce a detectar un aparente ciclo de 30 días en la OMJ sobre en norte de Suramérica, lo que podría sugerir que, por lo menos en el período analizado el modo cercano a los 60 días es menos marcado.

El cálculo del coeficiente de correlación de Pearson entre el IMJ120, IMJ80 con el IP5 de cada una de las estaciones del estudio arrojó valores muy bajos (entre 0,01 y 0,15 ) lo que podría estar sugiriendo que en el índice pentadal IP5 se registra el efecto de oscilaciones de mayor frecuencia que la de la OMJ. Por ello se trabajó con un IP quincenal (IP15) pretendiendo descartar tales oscilaciones de muy corto período. Aun así, los valores del coeficiente de correlación no variaron significativamente en comparación con lo ya obtenido en el análisis descrito para IP5, sin embargo, en la figura 3 en la que se compara el comportamiento de las series del IMJ80 con el IP15 de tres estaciones climatológicas situadas en tres regiones diferentes, es posible observar a simple vista un marcado ciclo de 30 días (en una quincena hay un máximo, en la siguiente un mínimo y así sucesivamente) en las dos series de datos, aunque en las de la IP15 es menos persistente esta frecuencia.

El análisis comparativo del comportamiento del IMJ y de los IP evidencian la existencia de un modo de 30 días en la escala intraestacional. Sin embargo, al correlacionar las series de los dos índices no se logra detectar relación alguna (los valores de los coeficientes son muy bajos). Esto, como ya se anotó puede estar ocurriendo debido a que en la precipitación hay más modos de variabilidad intraestacional que la generada por las ondas de 30-60 días de la OMJ. Una vía para explorar este aspecto es la comparación de los espectros del IMJ y de las series de los IPs.

Antes de comparar los espectros de la serie de IMJ con las de IPs, se elaboraron los espectros de ondita para la serie del MJI de $120^{\circ} \mathrm{W}$ y $80^{\circ} \mathrm{W}$, suavizadas con media móvil de 3 puntos, para el período 1994- 2004, con el fin de identificar las señales más significativas en el comportamiento de este índice. Estos espectros se presentan en la figura 4 en la que se puede ver que no hay diferencia entre los espectros de estos índices que presentan picos de potencia espectral a en la banda cercana a las 4-16 pentadas, es decir entre 20 y 80 días, además de resaltar las 128 y 512 pentadas, es decir 1,75 y 7 años, periodos correspondientes a la escala de la variabilidad interanual (cuasibienal y asociada al ciclo ENOS).

En la figura 5 se encuentra el análisis en onditas para las series del período 1994-2004 del índice pentadal de precipitación (IP5), suavizadas con medía móvil de 3 puntos, de 2 estaciones de subregiones de las principales regiones naturales de Colombia. La figura 5a muestran los espectros de onditas del IP5 para dos estaciones de la región Caribe y se puede, para las dos estaciones, una señal significativa sobre las pentadas 4-16, muy notoria en la pentada 8, lo que coincide con lo registrado en el espectro de IMJ (Figura 4). Para la región Andina (Figura 5b) los espectros de las series del IP5 de las dos estaciones (Neiva y Aeropuerto Eldorado de Bogotá) muestran igualmente señal significativa sobre la banda 4-8 pentadas (20 y 40 días) como el IMJ, aunque algunas señales cercanas a 16 pentadas (80 días) son igualmente notorias; también se percibe la variabilidad interanual con señales sobre 256-512 pentadas. En los espectros correspondientes a la región Pacífico (Figura 5c) se pueden observar dos máximos de la potencia espectral que sobresalen significativamente; estas señales están sobre períodos similares a los que se observan en la figura 4 que presenta los espectros del IMJ (4 
a)

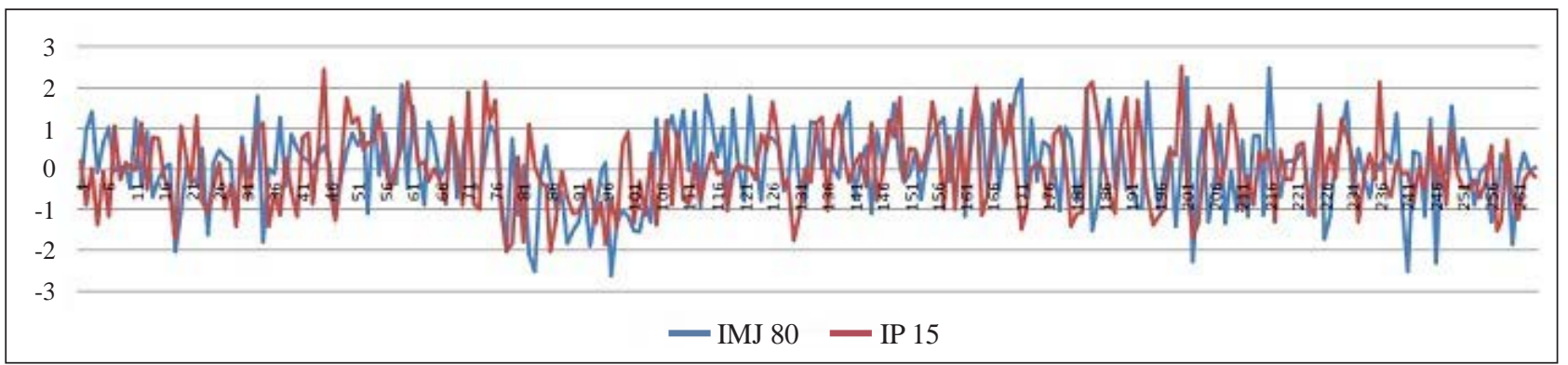

b)

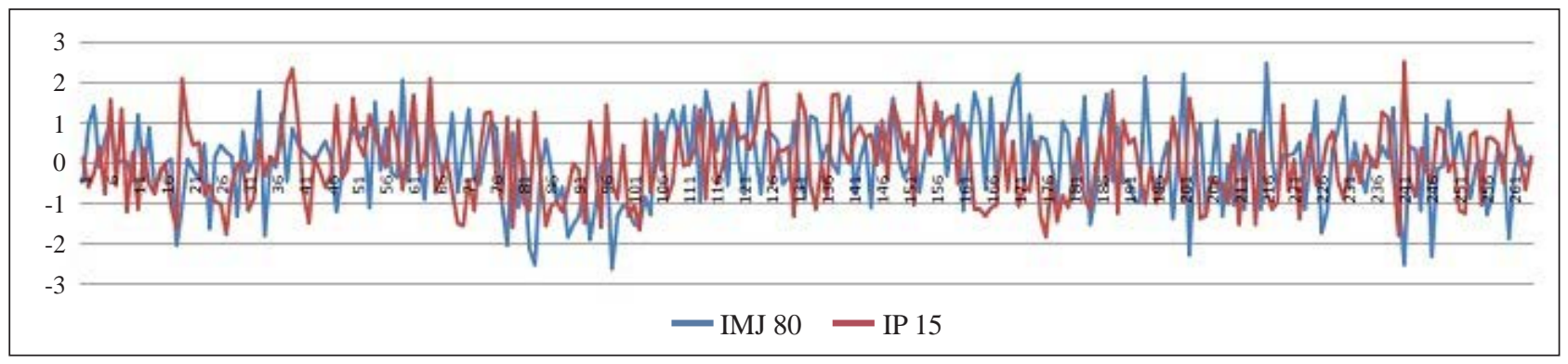

c)

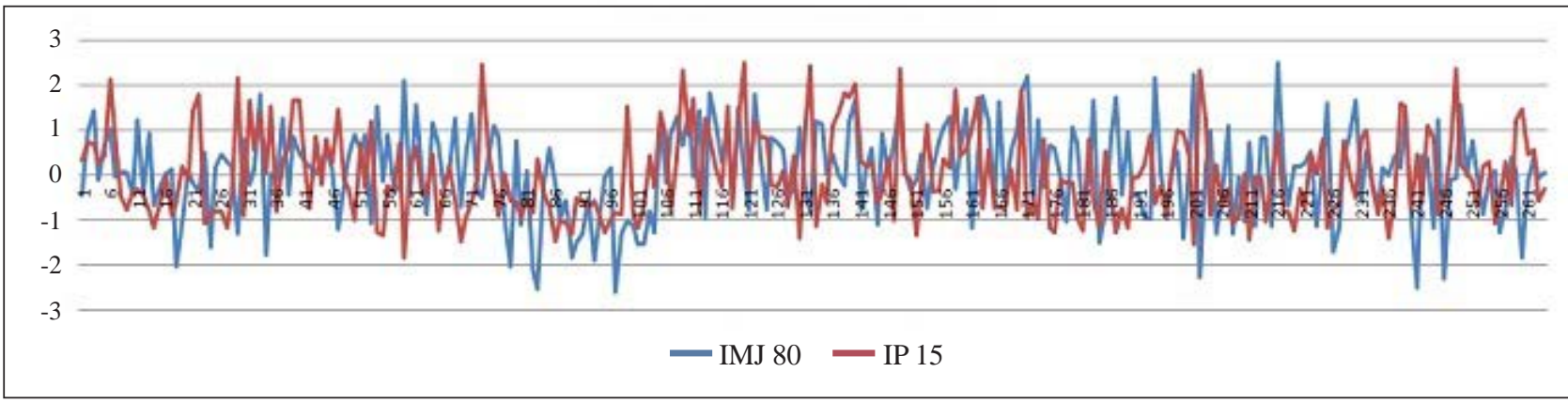

Figura 3. Análisis comparativo de las series del IMJ 80 y del IP15 de tres estaciones localizadas en las regiones Chocó (a), Antioquia (b) y Sabana de Bogotá (c) .

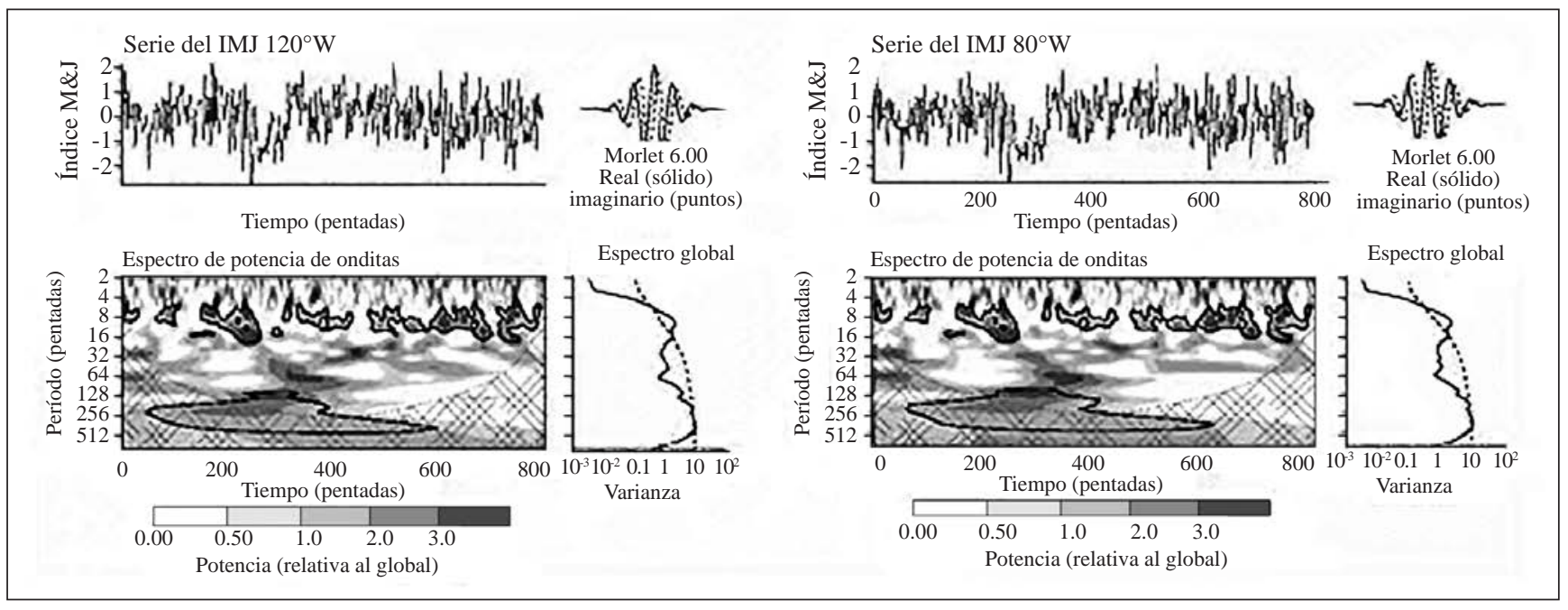

Figura 4. Espectro de potencia en onditas para IMJ 120W (a) y IMJ 80W (b) para la serie del periodo 1994-2004. 

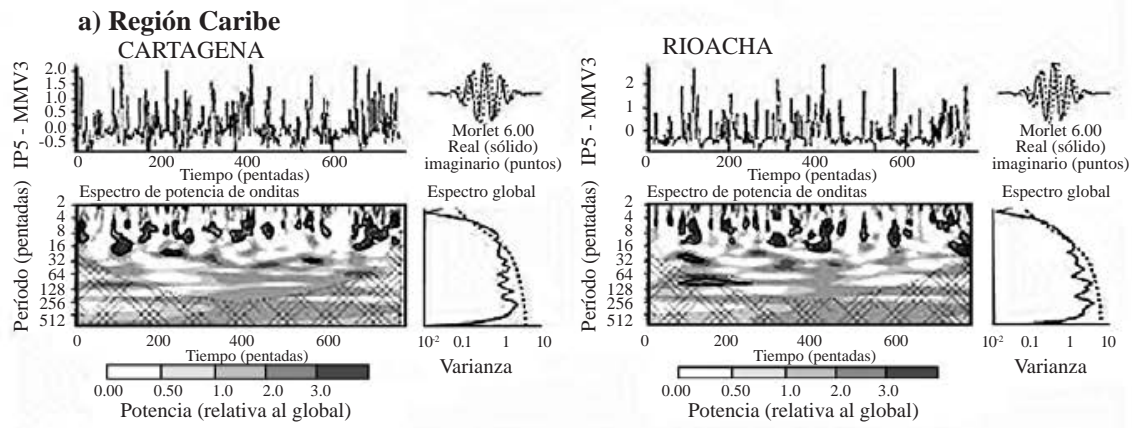

b) Región Andina

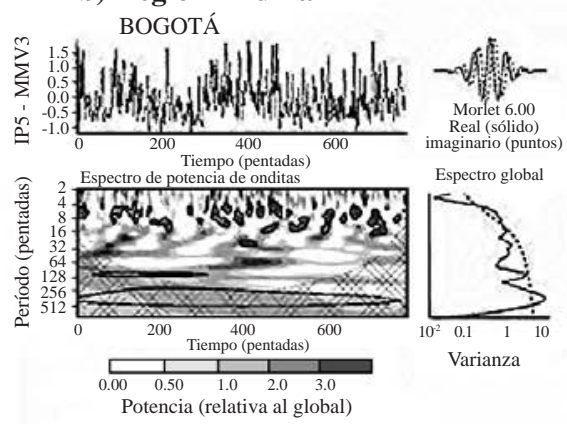

HUILA

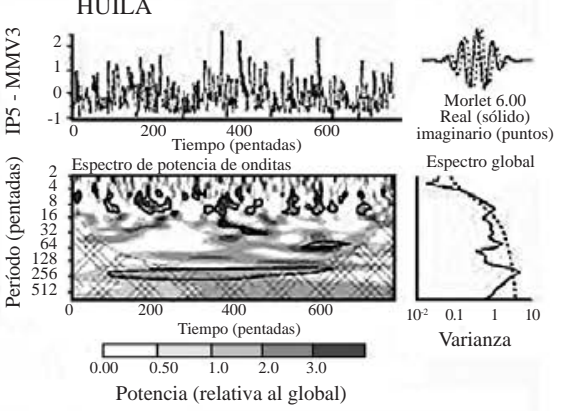

c) Región Pacífico
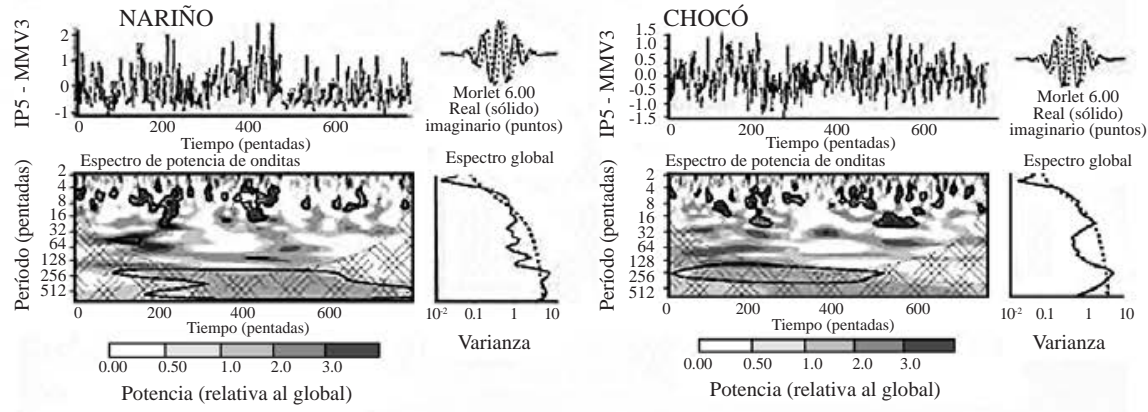

d) Región Orinoquía
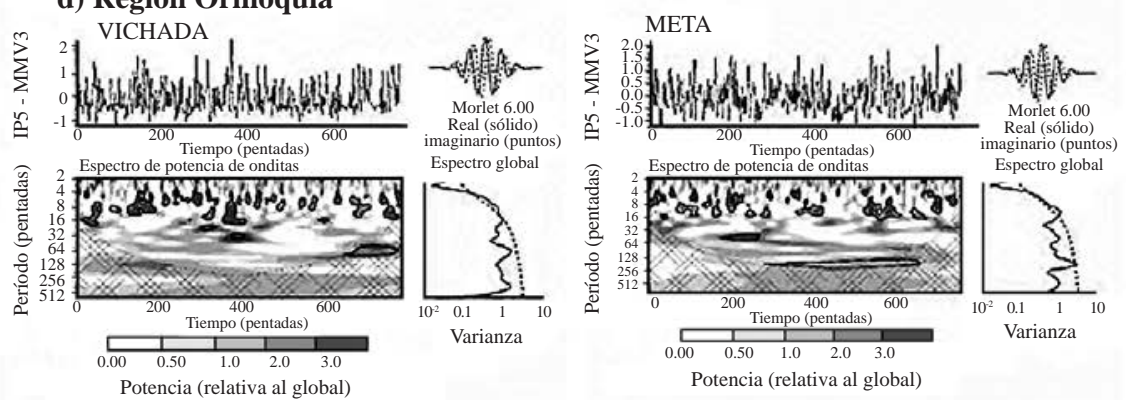

e) Región Amazonía
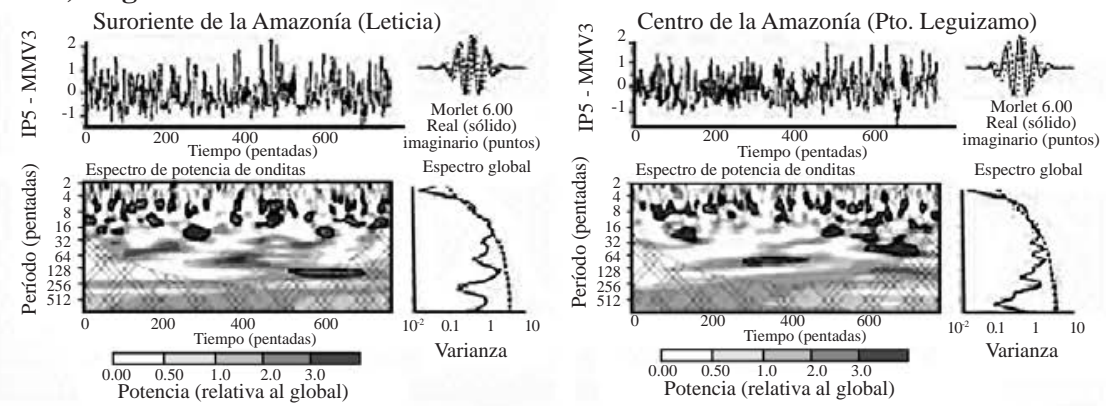

Figura 5. Espectro de potencia en onditas para las series del IP5 del período del periodo 1994-2004 de 12 subregiones de la región Caribe(a), Andina (b), Pacifico (c), Orinoquía (d) y Amazonía (e). 
y 16 pentadas o 20 y 80 días). Igualmente se puede notar la presencia de otras oscilaciones con periodos del orden de los 300 a 600 días, correspondientes a variabilidad interanuales de la precipitación; en Chocó, se vislumbra una señal importante sobre la pentada 30 (150 días aproximadamente). El espectro de onditas para el IP5 en estaciones de la Orinoquia (Figura 5d) resalta también la señal sobre las pentadas 4-16, marcada sobre la pentada 6 (aprox. 30 días). La figura 5e, que muestra espectros de onditas del IP5 para estaciones de la Amazonía, destaca señales significativas sobre la pentada 6 (o 30 días) y en el sector de las pentadas 8-16 (40-80 días).

El análisis espectral para las series de los índices decadales de precipitación (IP10), suavizadas con media móvil de tres puntos, se presenta en la figura 6. En las regiones Caribe (Figura 6a), Andina (Figura 6b) y Pacífica (6c) los espectros muestran picos significativos sobre el sector de las 4-8 décadas, es decir 40-80 días, que podrían se comparables con señales de variabilidad intraestacional. Para la Orinoquía (estaciones en Vichada y Meta en la Figura 6d), al igual que para la Amazonía (Caquetá y Leticia, en la Figura 6e) la señal destacada del espectro se presenta sobre la década 8 (80 días).

Los espectros de onditas de la series de IP quincenal (IP15) se presentan en la figura 7. Para todas las regiones la señal significativa se localiza entre las quincenas 2 y 4 que correspondería a períodos de 30-60 días. Se vislumbran otros picos, en la escala interanual, pero no sobresalen de la línea que representa el ruido rojo para considerarlos significativos.

Para el análisis del comportamiento de la precipitación de cada región bajo fases extremas de la OMJ, de las series del IMJ del período 1994-2004 se tomaron los 12 valores extremos (6 máximos con valores por encima de 2,0 y 6 mínimos con valores por debajo de -2,0) para la longitud de $120^{\circ} \mathrm{W}$ y se compararon con el IMJ en $80^{\circ} \mathrm{W}$, el índice pentadal de precipitación, la precipitación acumulada en la pentada y la velocidad vertical sobre el nivel de los $500 \mathrm{hPa}$. (Para minimizar el efecto de la variabilidad interanual, a la serie de IP5 se le aplicó una media móvil de 25 puntos, y esta se le resto a cada valor de la serie, obteniendo una nueva serie IP5* en la que las oscilaciones interanuales quedaron filtradas o por lo menos reducidas). En la tabla 1S, http:// www.raccefyn.co/index.php/raccefyn/article/download SuppFile/380/1811, se muestra el resultado del análisis para algunas de las 24 estaciones ubicadas a lo largo del territorio colombiano. En dicha tabla, en la primera columna se indica la fecha de la pentada en la que el IMJ-120 muestra el valor extremo, en la segunda columna se señala dicho valor extremo; en las siguientes columnas para cada valor (fila) del IMJ-120 se toman dos valores (2 filas) de IMJ-80 (el primer valor (primera fila) corresponde a lo observado en la misma pentada del IMJ-120 máximo o mínimo, el segundo valor (segunda fila) al de la pentada siguiente, esto teniendo en cuenta los 5 días que demora el recorrido de la fase de la $\mathrm{MJO}$ de $120^{\circ} \mathrm{W}$ a $\left.80^{\circ} \mathrm{W}\right)$, de índice de precipitación
(IP5*), de precipitación pentadal (PPT, en milímetros) y de velocidad vertical promedio de la pentada del extremo de OMJ (VVEL, en $\mathrm{hPa} / \mathrm{s}$ ).

En la tabla $1 S$ se puede observar la diversidad de la respuesta a las fases extremas de la OMJ en diferentes regiones del país. Los movimientos verticales no presentan un patrón de respuesta definido, en tanto que el IP5* (resaltado en escala de colores correspondientes a intervalos del mismo) tiende a diferenciarse en su respuesta a las fases extremas de la OMJ, aunque en algunos casos la diferencia no es perceptible. Por ejemplo, en las regiones Caribe y Andina, la fase negativa (en los valores más bajos) tiende a propiciar lluvias abundantes y la fase positiva (en los máximos) a desestimular la precipitación, respuesta que se evidenció en estaciones localizadas en Montería, Riohacha, Santa Marta, Cartagena y San Andrés; para Cesar la respuesta se presenta al contrario: lluvias abundantes en la fase positiva y disminución de precipitación en la negativa.

En el sector sur del Pacífico colombiano (Nariño y Cauca) a los máximos del IMJ corresponde en general desactivación de la precipitación, a los mínimos - reactivaciones de la misma; pero en Chocó esta relación no es clara. En la Orinoquía, en el piedemonte llanero hay una relación clara: máximos corresponde a incremento de la precipitación, mínimos - reducción; sin embargo, al norte, en Arauca aunque se vislumbra el misma tipo de respuesta, para el período de análisis se registró un evento de incremento extremo de lluvias bajo la fase positiva. Para la región amazónica no resulta clara la señal de respuesta de la precipitación a las fases extremas de la OMJ.

La respuesta de la precipitación de las regiones a las fases extremas se sintetizó en los mapas de la figura 8, los cuales pueden ser utilizados para predicción climática a un mes ya que conociendo la fase que dominaría sobre el territorio colombiano en el mes siguiente se podría anticipar si habría activación o receso de las lluvias.

La figura 9 presenta una prueba del esquema de predicción (mapas de la Figura 8) para el periodo desde junio de 2012 hasta noviembre de 2012 donde se perciben 2 periodos con fases extremas de la OMJ; una fase negativa (mínimos del IMJ) hacia mediados de junio y una fase positiva (máximos del IMJ) para la primera quincena de septiembre. Estos periodos se compararon con el esquema propuesto por el trabajo (en la columna vertical al centro) y con los mapas de anomalías de la precipitación presentados para estos meses (mapas de la columna de la derecha). Para la fase mínima observada en junio de ese año, el esquema de predicción señala amplias zonas con aumento de la precipitación, especialmente para sectores de Caquetá y Cauca y amplios sectores de la región Caribe, Norte de Santander, Meta y Cauca con disminución de las lluvias, sin embargo, al observar el mapa de anomalías de precipitación para junio se advierte que en amplios sectores del territorio colombiano se presentaron anomalías negativas de la precipitación, aunque el mapa de predicción y el de anomalías reales coinciden en sectores con anomalías 
a) Región Caribe

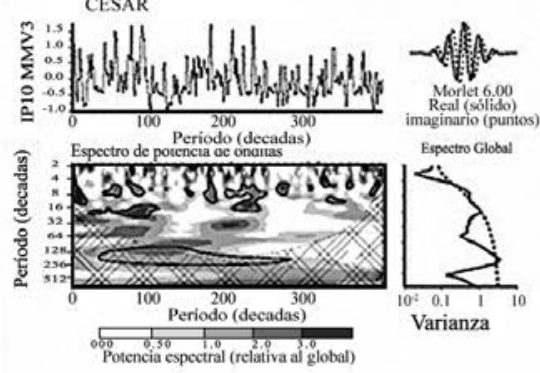

b) Región Andina

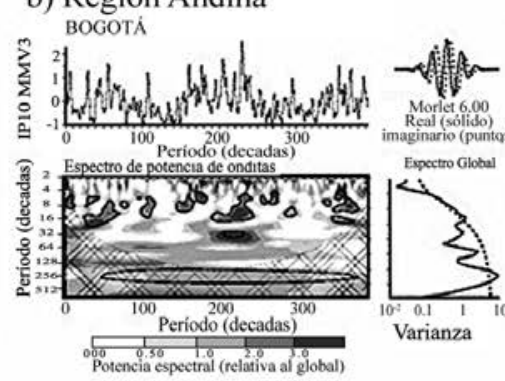

c) Región Pacífico CHOCó

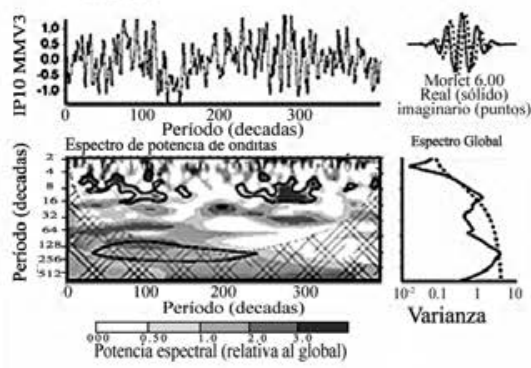

d) Región Orinoquía VICHADA

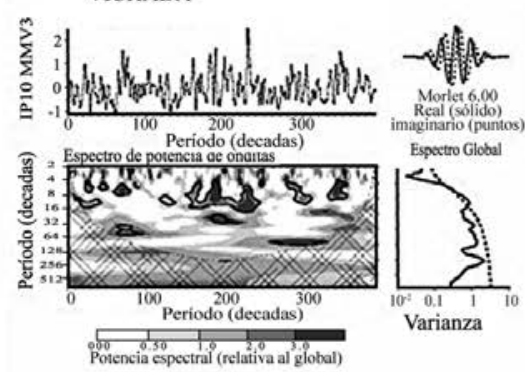

e) Región Amazonía CAQUETÁ
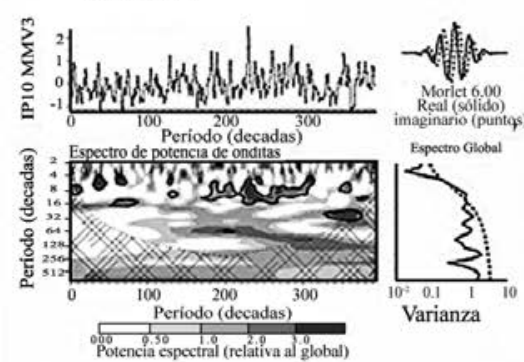
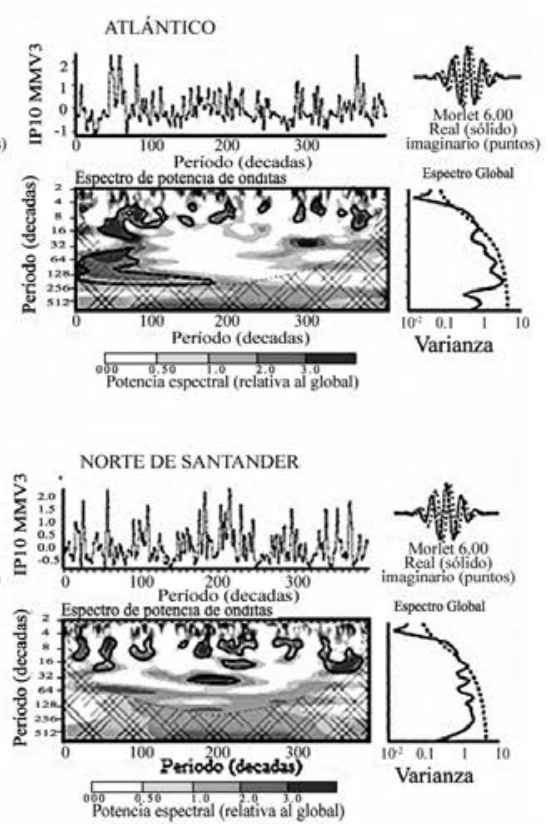

NARIÑO

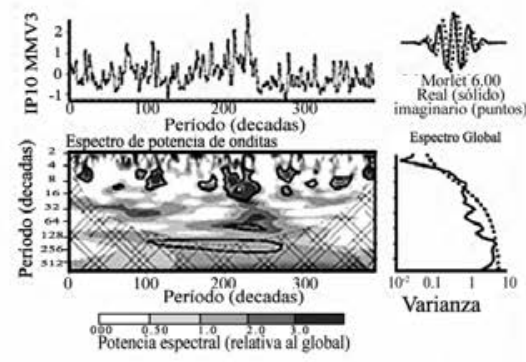

META
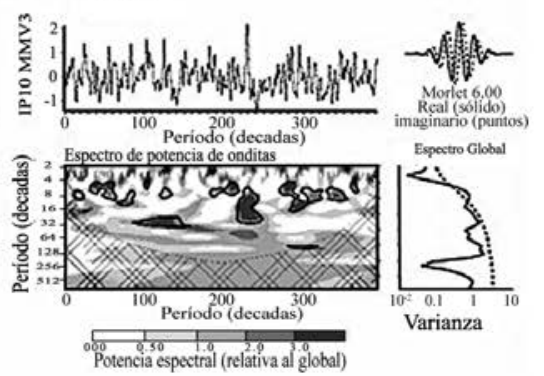

AMAZONAS
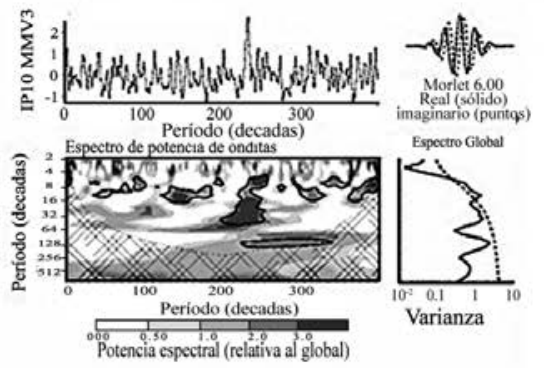

Figura 6. Espectro de potencia en onditas para las series del IP10 del período del periodo 1994-2004 de 12 subregiones de la región Caribe(a), Andina (b), Pacifico (c), Orinoquía (d) y Amazonía (e). 
a) Región Caribe
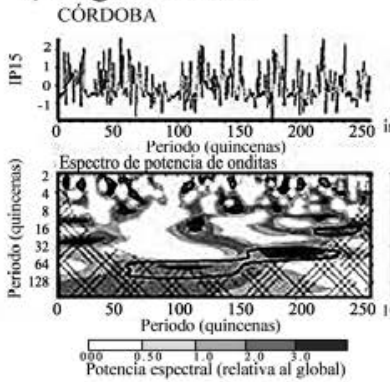

b) Región Andina
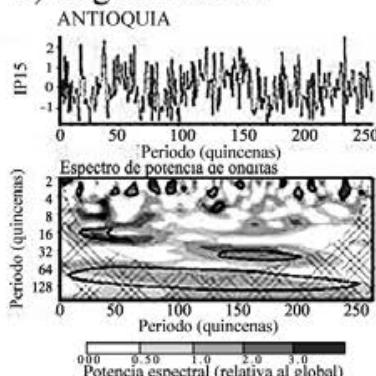

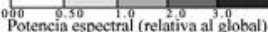

c) Región Pacífico
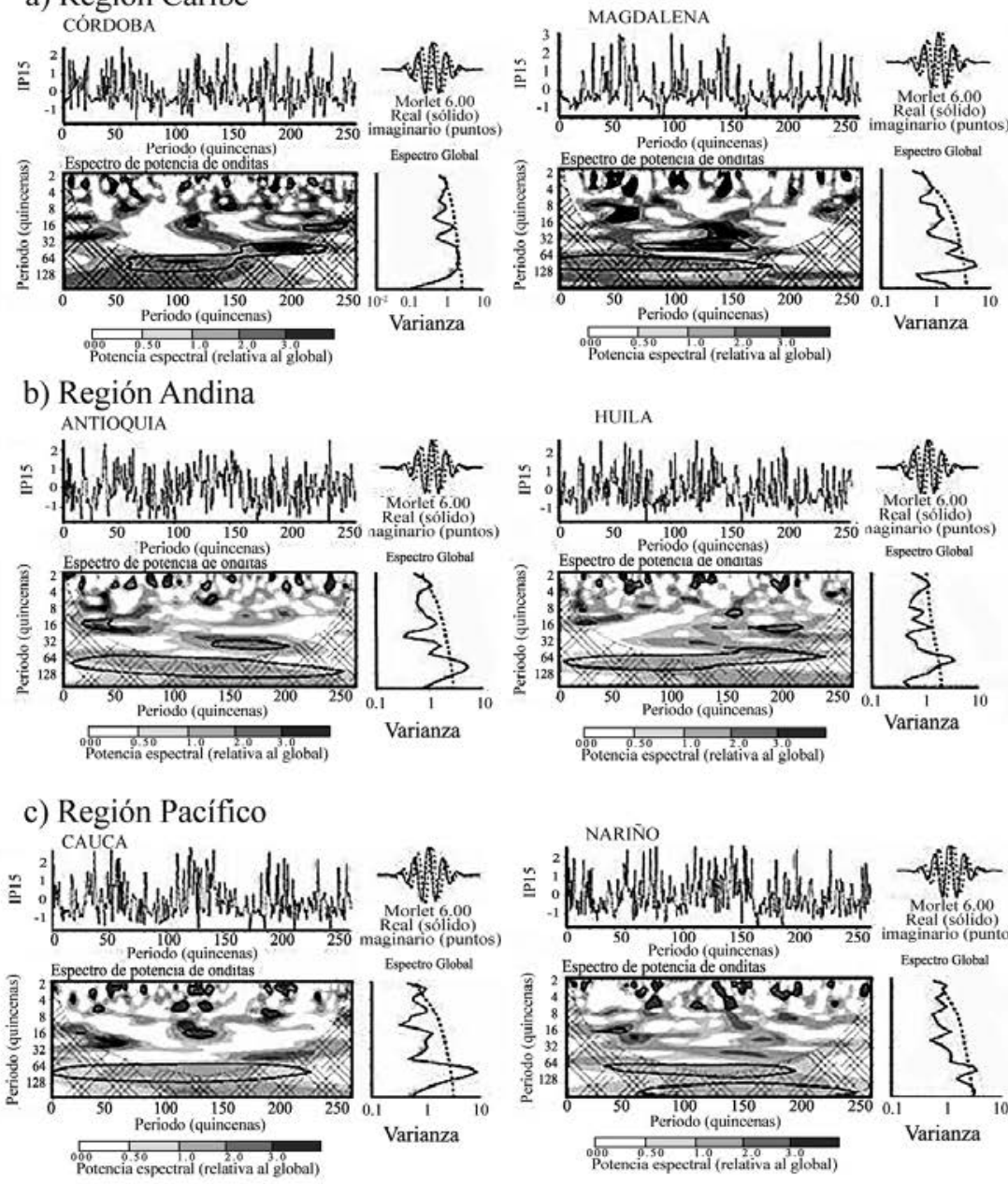

HUILA
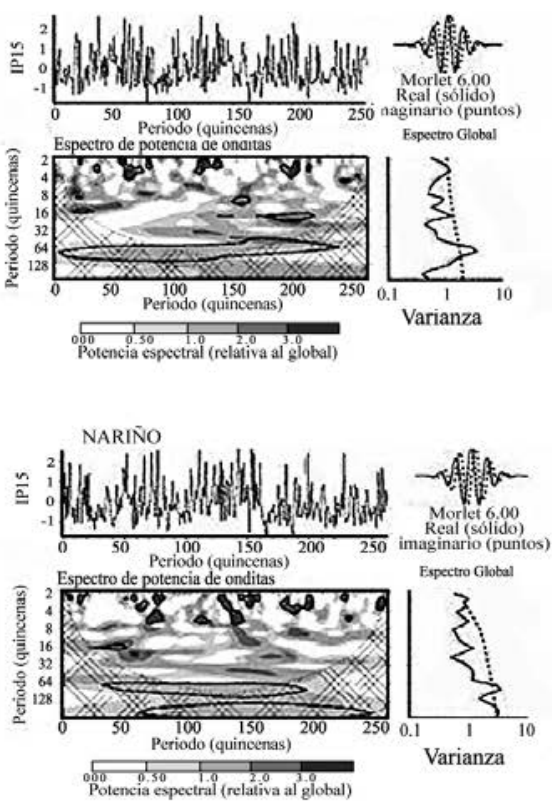

d) Región Orinoquía
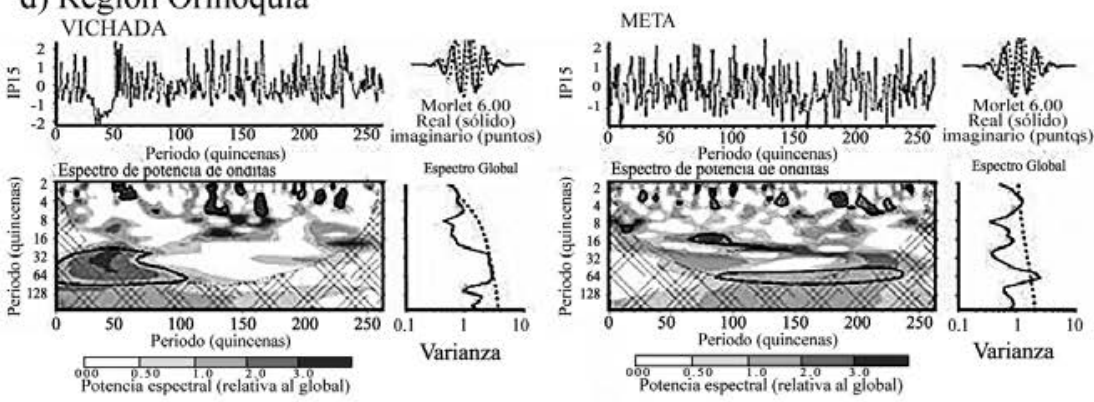

e) Región Amazonía
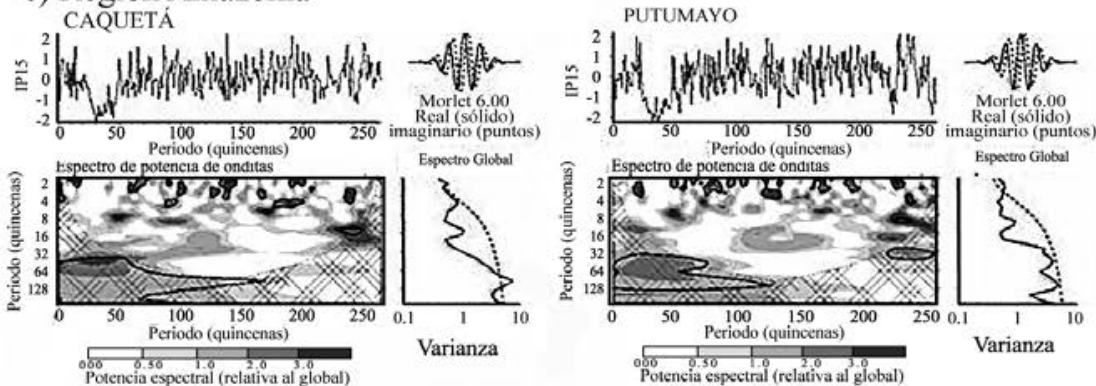

Figura 7. Espectro de potencia en onditas para las series del IP15 del período del periodo 1994-2004 de 12 subregiones de la región Caribe(a), Andina (b), Pacifico (c), Orinoquía (d) y Amazonía (e). 


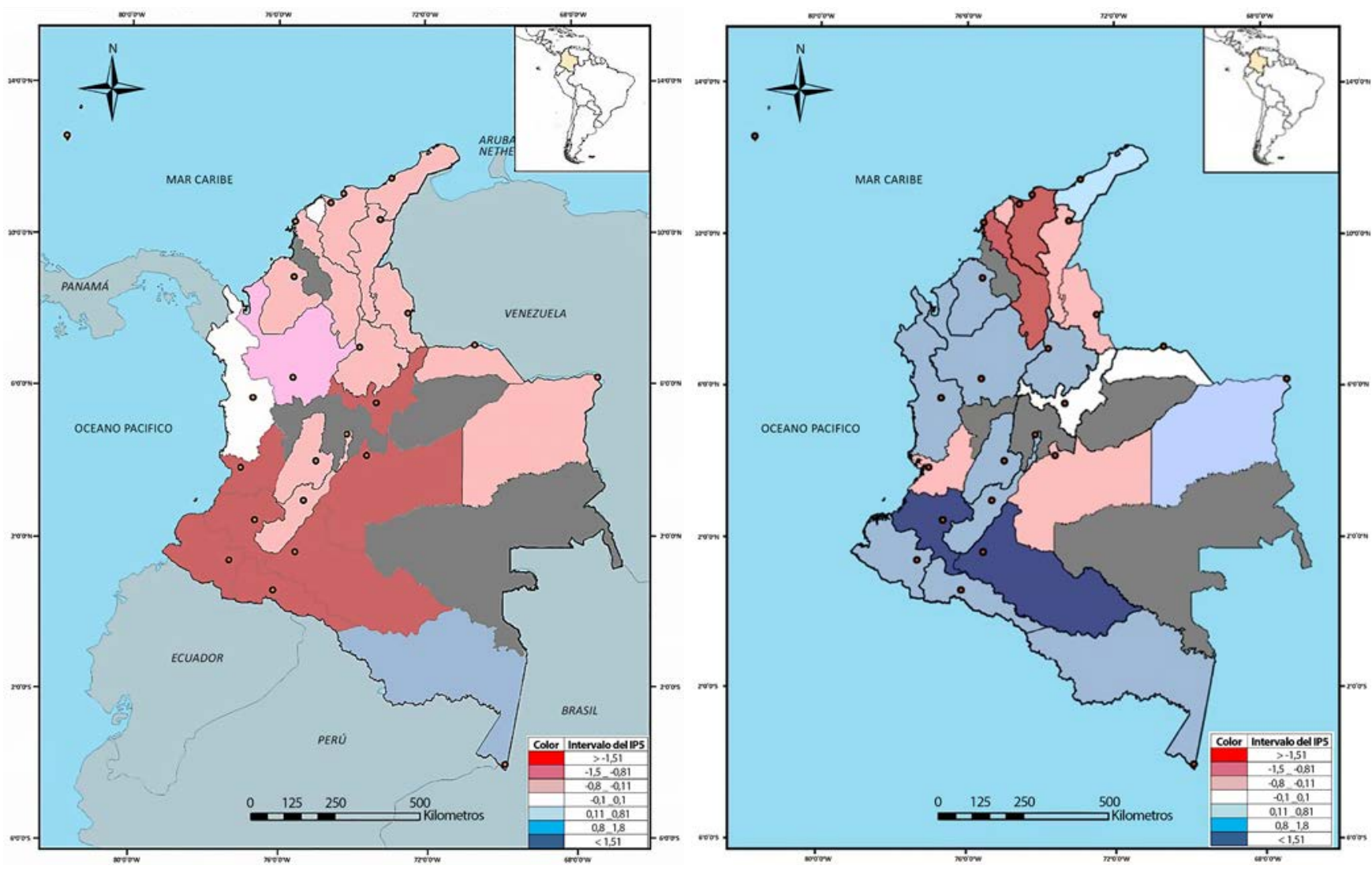

Figura 8. Respuesta del IP5* en diferentes regiones del territorio colombiano cuando la fase máxima (izquierda) o mínima (derecha) del IMJ está en $120^{\circ} \mathrm{W}$. (Los colores rojo y rosado señalan disminución de la precipitación; los tonos de azul - aumento; el gris señala las regiones donde la señal no es clara hacia aumento o disminución.

positivas de precipitación especialmente hacia Amazonas, Córdoba, Santander y Chocó; también concuerdan las anomalías negativas de precipitación en sectores de la Región Caribe y Valle. Para la fase máxima del IMJ observada en la primera quincena de septiembre, el mapa de predicción indica una posible disminución de las lluvias a lo largo del territorio colombiano, exceptuando, sectores de Amazonas, Chocó y Atlántico; el mapa de anomalías mensuales de septiembre muestra anomalías negativas a lo largo del país, con algunas anomalías positivas sobre el oriente de la Orinoquia, Amazonas y Sur de la Región Caribe, situación que concuerda ampliamente con lo mostrado por el mapa de predicción para la fase máxima del IMJ.

\section{Conclusiones}

El análisis espectral del IMJ en $120^{\circ} \mathrm{W}$ y en $80^{\circ} \mathrm{W}$ permitió corroborar que para la región del norte de Suramérica el componente de 30 días de la OMJ se presenta más marcado que el de 60 o cualquier otro de la escala intraestacional, lo que en gran medida corrobora lo identificado en la exploración inicial.

El análisis espectral de las series de índice de precipitación pentadal, decadal y quincenal muestra claramente una señal sobre los 30-40 días que corresponde a la escala intraestacional. También se hicieron evidentes señales de la variabilidad interaunal, pero la potencia de su espectro fue menor y con bajo grado de significancia, debido tal vez a que estas oscilaciones tienen menor amplitud que las intraestacionales.

El intento por cuantificar una relación entre el Índice de Madden-Julian y los índices de precipitación, toda vez que se encontró una señal común bastante marcada sobre el período de los 30-40 días, no dio resultados satisfactorios: los coeficientes de correlación fueron muy bajos. Tal vez esto se debió a la presencia de otras señales, aunque poco significativas en los espectros, que no fueron filtradas, actúan a manera de ruido y dificultan la visibilización de la relación.

El análisis de la respuesta de la precipitación regional (representada por los IP) ante fases extremas de las oscilaciones Madden-Julian (representadas por el IMJ) dio como resultado que bajo extremos positivos de la IMJ (fase positiva o subsidente) se produce disminución de la precipitación pentadal en algunas regiones del país; con valores extremos negativos de la IMJ (fase negativa o convectiva) ocurre aumento de la precipitación pentadal en varias regiones; para siete de las 24 regiones no se identificó un patrón de respuesta definido. 


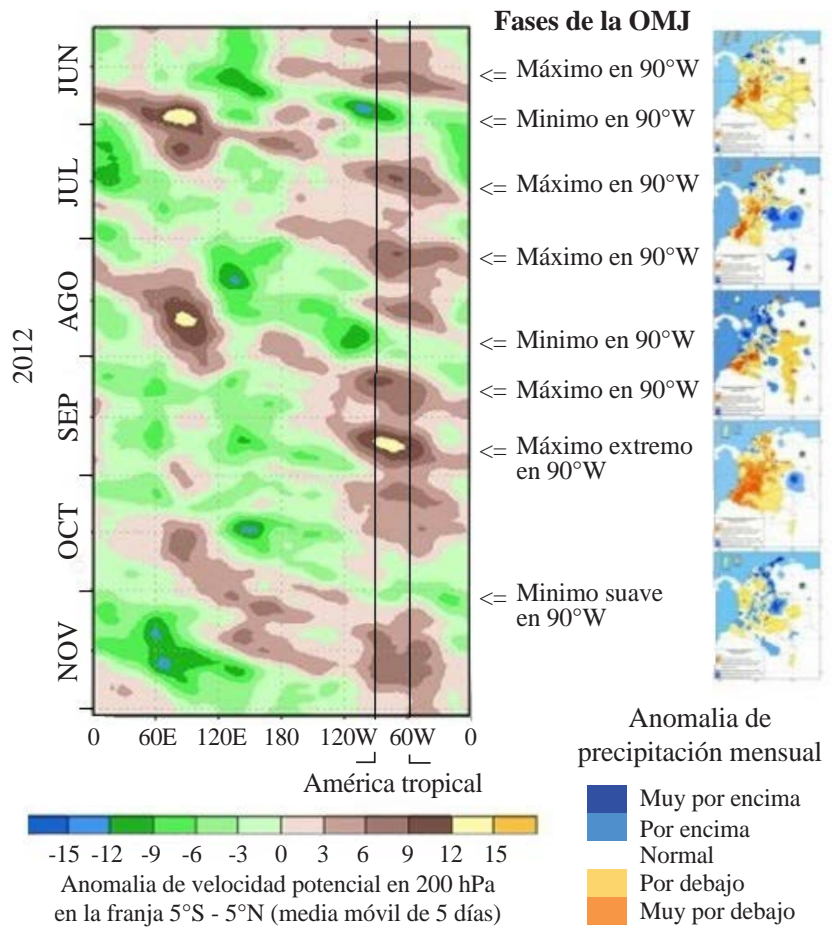

Figura 9. Comprobación de los mapas de predicción de respuesta de la precipitación de diferentes regiones de Colombia a fases extremas de OMJ. En la columna de la izquierda se muestra el comportamiento de la anomalía de velocidad potencial en $200 \mathrm{hPa}$; en la columna vertical del centro se señalan las fases extremas de IMJ que indicarían cual mapa-esquema de la Figura 8 se utilizaría para la predicción; en la columna de la derecha los mapas de anomalías de la precipitación mensual obtenidos de los informes del IDEAM.

Sobre la base de la relación establecida entre los extremos de la IMJ y la IP pentadal en diferentes regiones del territorio colombiano, se elaboró un mapa que sirve de base para la predicción climática en la escala intraestacional, el cual se comprobó con buenos resultados en cuanto a la fase positiva y parcialmente satisfactorios para la fase negativa.

\section{Información suplementaria}

Tabla 1S. Eventos extremos de la OMJ representados por valores de IMJ mayores que 2.0 o menores que -2.0 y los respectivos valores del IP5, la precipitación pentadal y la velocidad vertical en $500 \mathrm{hPa}$ en diferentes estaciones de las regiones analizadas. Vea la tabla 1S en: http://www.raccefyn.co/index.php/raccefyn/article/ download SuppFile/380/1811

\section{Agradecimientos}

El presente trabajo se desarrolló en el marco del proyecto "Efecto de las ondas Madden-Julian en la precipitación sobre regiones del territorio colombiano” apoyado por la Dirección de Investigación de la Sede Bogotá (DIB) de la Universidad Nacional de Colombia bajo el código No. 14522, dentro de la línea de "Impacto socioeconómico de la variabilidad climática" que desarrolla el grupo de investigación “Tiempo, clima y sociedad” del Departamento de Geografía, Facultad de Ciencias Humanas, de la Universidad Nacional de Colombia. Los autores agradecen al Instituto de Hidrología, Meteorología y Estudios Ambientales IDEAM por facilitar los datos de las estaciones utilizadas en el análisis que se expuso en el presente trabajo.

\section{Conflicto de intereses}

Los autores declaran que no tienen conflicto de intereses.

\section{Referencias}

Álvarez, M.S., Vera, C.S., Kiladis, G.N., Liebmann, B. (2016). Influence of the Madden-Julian Oscillation on precipitation and surface air temperature in South America. Climate Dynamic, 46 (1). doi: 1007/s00382-015-2581-6

Carvalho, L., Jones, L. (2004). The South Atlantic Convergence Zone: Intensity, form, persistence and relationships whit intraseasonal to interannual activity and extreme rainfall. J. Climate. 17: 88-108.

Cuadros, N. (2010). Análisis de la variabilidad de la temperatura del aire en regiones de Colombia bajo la influencia de la oscilación Madden-Julian durante los años 1978-2008. Bogotá: Unversidad Nacional de Colombia.

Donald, A., H. Meinke, B. Power, A. de H. N. Maia, M. C. Wheeler, N. White, R. C. Stone, and J. Ribbe (2006). Near-global impact of the Madden-Julian Oscillation on rainfall, Geophys. Res. Lett. 33: L09704, doi: 10.1029/2005GL025155

Dorado, J. (2007). Tesis de Maestría, Programa de Postgrados en Meteorología, Departamento de Geociencias, Facultad de Ciencias, Universidad Nacional de Colombia.

Hurtado, G. (2000). La precipitación en Colombia. Nota técnica IDEAM, No. IDEAM/METEO/006/2000. Bogotá. 30 p.

Janicot, S., Sultan, B. (2001). Intraseasonal modulation of convection in the West Africa monsoon. Geophys Res Lett., 28 (3): 523-526.

Jones, C., Schemm, J-E. E. (2000). The influence of Intraseasonal Variations on Medium- to Extended-range Weather Forecasts over South America. Monthly Weather Review, 128 (2): 486-494.

Krishnamurti, V., Shukla, J. (2007). Intraseasonal and Seasonally persisting patterns of Indian Monsoon rainfall. J. of Climate, 20 (1), 3-20.

Lau, W., \& Waliser, D. (2012). Intraseasonal Variability in the atmosphere-ocean climate system. Chichester. SpringerPraxis Books in Environmental Sciences. Berlin-Heidelberg, 613p.

Madden, R., Julian, P. (1971). Detection of 40-50 day oscillation in the zonal wind in the tropical Pacific. J, Atmos. Sci. 28 (7): 702-708.

Martin, E., Schumacher C. (2012). Modulation of Caribbean precipitation by the Madden-Julian Oscillation. Journal of Climate, 813-824.

NOAA, (2010). Climate Prediction Center (CPC). Recuperado el 12 de Octubre de 2010, de http://www.cpc.noaa.gov/ products/precip/CWlink/daily_OMJ_index/proj_norm order.ascii. 
Paegle, J.N., Byerle, L.A., Mo, K.C. (2000). Intraseasonal Modulation of South American Summer Precipitation. Monthly Weather Review. 128 (3): 837-850

Pabón, J., J. Dorado, (2008). Intraseasonal variability of rainfall over northern South America and Caribbean. Earth Sci Res J., v. 112: 194-212.

Pabón, J.D. (2011). Improving Climate Prediction Schemes with Intraseasonal Variability: A Key Tool toward Hydrometeorological Disasters Reduction in Tropical America. In: "Natural Disasters Policy Issues and Mitigation Strategies" (Andi Eka Sakya, (Ed.), Centre for Science \& Technology of the Non-Aligned and other Developing Countries (NAM S\&T Centre), New Delhi, India, 229p), 127-137.

Poveda, G., Mesa, O., Agudelo, P., \& Alavarez, J. (2002). Influencia del ENSO, oscilación Madden-Julian, ondas del este, huracanes y fases de la luna en el ciclo diurno de precipitación en los Andes Tropicales de Colombia. Meteorología Colombiana. 5: 3-12.

Torrence, C., Compo, G. (1998). A practical Guide to Wavelet Analysis. Bull. Of Am. Meteo. Soc. 79 (1): 61-78.

Torres-Pineda C. E. (2012). Efecto de las ondas Madden-Julian en la precipitación sobre algunas regiones del territorio colombiano. Tesis de Maestría, Programa de Postgrado en Meteorología, Departamento de Goeciencias, Universidad Nacional de Colombia, Bogotá, Colombia. 80 páginas.

Trickey, M., Cornejo, J. (2012). Evaluación de la oscilación Madden-Julian en la predicción de niveles de la costa ecuatoriana. Aqua-Lac. 2 (1): 37-44.

Wang, X., Zhang, X. (1996). Intraseasonal oscillation and associated spatial-temporal structures of precipitation over China. J. of Geophysical Research. 101: 19035-19042.

Waliser, D.E., Lau, K.M,. Stern, W, Jones, C. (2003). Potential Predictability of the Madden-Julian Oscillation. Bull. Amer. Meteor. Soc., 84: 33-50. doi: http://dx.doi.org/10.1175/ BAMS-84-1-33

Ye, H., Cho, H. (2001). Spatial and temporal characteristics of intraseasonal oscillation of precipitation over the United States. Theorical and Appled Climatology, 68 (1): 51-66.

Yepes, J., Poveda, G. (2013). Diagnóstico y predictabilidad de la lluvia en Colombia a escala intraestacional. Revista Colombia Amazónica, 6: 17-29.

Zhang, C. (2013). Madden-Julian Oscillation. Bridging Weather and Climate. Bull. Amer. Meteor. Soc. 94 (12), pp. 18491870. doi: http://dx.doi.org/10.1175/BAMS-D-12-00026.1 